INPLASY

PROTOCOL

To cite: Ji et al. Prior bariatric surgery is a potential protective factor against severe COVID-19: A systematic review and metaanalysis. Inplasy protocol 202210077. doi: 10.37766/inplasy2022.1.0077

Received: 16 January 2022

Published: 16 January 2022

Corresponding author:

Yuedong Wang

2112014@zju.edu.cn

Author Affiliation:

The Second Affiliated Hospital, School of Medicine, Zhejiang University.

Support: None.

Review Stage at time of this submission: Preliminary searches.

Conflicts of interest:

None declared.

\section{Prior bariatric surgery is a potential protective factor against severe COVID-19: A systematic review and meta-analysis}

Ji, Y1; Zhu, J2; Wang, Y33.

Review question / Objective: We aimed to investigate any potential correlation between history of bariatric surgery and improved outcomes in COVID-19 patients.

Condition being studied: Obesity and several obesity-related diseases are risk factors for severe COVID-19. Bariatric surgery successfully treats obesity-related conditions. The aims of our study are to review existing clinical evidence and compare the rates of mortality, use of invasive mechanical ventilation, and ICU admission in patients hospitalized for COVID-19 with or without a history of bariatric surgery.

Information sources: Systematic literature search will be performed in Medline, Embase, Cochrane, and Scopus database.

INPLASY registration number: This protocol was registered with the International Platform of Registered Systematic Review and Meta-Analysis Protocols (INPLASY) on 16 January 2022 and was last updated on 16 January 2022 (registration number INPLASY202210077).

\section{INTRODUCTION}

Review question / Objective: We aimed to investigate any potential correlation between history of bariatric surgery and improved outcomes in COVID-19 patients.
Condition being studied: Obesity and several obesity-related diseases are risk factors for severe COVID-19. Bariatric surgery successfully treats obesity-related conditions. The aims of our study are to review existing clinical evidence and compare the rates of mortality, use of 
invasive mechanical ventilation, and ICU admission in patients hospitalized for COVID-19 with or without a history of bariatric surgery.

\section{METHODS}

Participant or population: COVID-19 patients with or without a history of bariatric surgery.

Intervention: With a history of bariatric surgery.

Comparator: Without a history of bariatric surgery.

Study designs to be included: We will include observational studies.

Eligibility criteria: We will include peerreviewed studies that compared the rates of mortality, use of invasive mechanical ventilation, and ICU admission in COVID-19 patients with and without a history of bariatric surgery.

Information sources: Systematic literature search will be performed in Medline, Embase, Cochrane, and Scopus database.

Main outcome(s): Mortality, use of invasive mechanical ventilation, and ICU admission.

Quality assessment / Risk of bias analysis: The methodological quality of nonrandomized studies was evaluated using the Newcastle-Ottawa quality assessment scale.

Strategy of data synthesis: Data on dichotomous outcomes are expressed as odds ratio (OR) with $95 \%$ confidence intervals (Cl). Statistical heterogeneity was assessed using the 12 methodology.

Subgroup analysis: None.

Sensitivity analysis: If 12 was > $50 \%$, we performed a sensitivity analysis by removing 1 study at a time (guided by highest 12) until the sensitivity was below the threshold of $50 \%$.
Country(ies) involved: China.

Keywords: Bariatric surgery, COVID-1 9, Meta-analysis, Mortality.

Contributions of each author:

Author 1 - Yun Ji.

Email: yunji@zju.edu.cn

Author 2 - Jinhui Zhu.

Email: 2512016@zju.edu.cn

Author 3 - Yuedong Wang.

Email: 2112014@zju.edu.cn 